\title{
COMPORTAMENTO DE CITAÇÃO NO CAMPO DA CANCEROLOGIA BRASILEIRA
}

\author{
Kátia de Oliveira Rodrigues \\ Doutoranda em Ciência da Informação \\ Programa de Pós-Graduação em Ciência da Informação \\ Universidade Federal de Minas Gerais \\ katiarodrigues10@gmail.com \\ Marlene Oliveira \\ Doutora em Ciência da Informação \\ Professora do Programa de Pós-Graduação em Ciência da Informação \\ Universidade Federal de Minas Gerais \\ marleneotmelo@gmail.com
}

\section{Resumo}

$\mathrm{O}$ artigo tem como objetivo caracterizar o comportamento de citação dos docentes-pesquisadores do campo da Cancerologia brasileira, através da análise das referências elencadas por estes em suas produções científicas publicadas no período de 2005-2015. A amostra foi constituída de 1.016 citações. Os resultados sugerem que, no campo em análise, o artigo é a principal tipologia das produções citadas entre os docentes-pesquisadores, correspondendo a $80 \%$ das citações. O comportamento de citação observado em relação à autoria múltipla se reproduz em todo o período analisado, com ocorrência superior ao de autoria individual, $936(92,1 \%)$ e 80 (7,9\%), respectivamente. Em relação ao idioma das citações, o inglês corresponde a $1005(99,0 \%)$ das citações.

\section{Palavras-chave}

Análise de citação. Cancerologia brasileira. Comportamento de citação.

\section{INTRODUÇÃO}

Nos primórdios do fazer científico, os resultados das pesquisas eram disseminados por cartas manuscritas ou impressas, conforme a quantidade de pesquisadores que se pretendia alcançar (MEADOWS, 1999). Do ponto de vista dialógico, as cartas representavam uma alternativa positiva, tendo em vista que eram direcionadas aos pesquisadores que discutiam temas semelhantes ou próximos. Exemplo dessa comunicação informal é relatado pela pesquisadora brasileira Freire (1996, p. 4), ao abordar a troca de carta entre Hersche ${ }^{1}$ e Lyell': "sua carta [a de

\footnotetext{
${ }^{1}$ John Frederick William Herschel foi um cientista de referência; nascido em 1792, faleceu em 1871, aos 79 anos. Recebeu vários prémios, entre eles a Medalha de Ouro da Royal Astronomical Society.
}

Herschel] a Lyell sobre o 'mistério dos mistérios' tornou-se conhecida, pois Lyell a fez circular entre os cientistas [...]".

Contudo, se, por um lado, a troca de cartas entre cientistas era positiva para o avanço da ciência, por outro, o encaminhamento das cartas para um grupo específico de pesquisadores poderia ser negativo, uma vez que os "[...] autores quase nunca as enviavam para aqueles que podiam refutar suas teorias ou rejeitar seus exemplos", como explica Stumpf (1996, p. 1). A ação de encaminhar as cartas para determinados pesquisadores poderia fragilizar um dos objetivos, não o único, da comunicação científica, que consiste em validar ou não validar os

\footnotetext{
2 Charles Lyell, cientista e advogado, nasceu em 1797 e faleceu em 1875, amigo e grande influenciador da Teoria Evolucionista de Charles Darwin.
} 
resultados de uma pesquisa em função do atendimento a certas regras da área.

Com a invenção da prensa de tipos móveis de Gutemberg, na Europa do século $\mathrm{XV}$, os resultados das pesquisas também se tornaram impressos - na forma de carta, livro e atas, permitindo que um número maior de pesquisadores tivesse acesso. Alguns desses impressos apresentavam o formato de livros, a exemplo do livro de Astronomia moderna - De revolutionibus orbium coelestium, de Copérnico, em 1543, e de Anatomia humana - De humani corporis fabrica, de Vesálio. Utilizando-se do serviço de correio existente na Europa, os livros eram entregues à comunidade científica. A relevância do livro para disseminação dos resultados da pesquisa foi significativa; entretanto, Meadows (1999) adverte que o periódico científico surge no século XVII, para expandir e, em alguns casos, substituir os canais formais de comunicação - livro, carta, entre outros.

Durante o processo de comunicação científica, os resultados das pesquisas podem ser avaliados e aceitos ou não pelos pares, avaliação essa que pode ou não lhes conferir status de cientificidade (GARVEY, 1979, MUELLER, 2007). Sobre a aprovação dos resultados de pesquisa, Mueller (2007) complementa que ela ocorre tanto durante a submissão para publicação, como após a mesma. A segunda etapa da avaliação, a aceitação por parte dos pares, ocorre quando os resultados de uma determinada pesquisa, ao serem publicados, servem de ponto de partida para novas pesquisas, configurando assim a prática de citação. Essa prática, como objeto de estudo no campo da comunicação científica, tem muito a oferecer para a compreensão e o delineamento de determinadas áreas do conhecimento, no que se refere aos aspectos de sua produtividade e comportamento de uso da informação científica registrada.

$\mathrm{Na}$ literatura científica brasileira há estudos sobre análise de citação. Estes estudos se dispersam em diferentes campos. No campo da Cancerologia, os estudos sobre citações são escassos. Um exemplo de trabalho nesse campo é o de Nieder et al. (2015), que pesquisaram o impacto dos artigos pu- blicados em dois periódicos científicos do campo: o Radiation Oncology Investigations e o Journal of Radiosurgery.

A pesquisa da qual o presente artigo apresenta um recorte nasce, pois, da tentativa de colaborar com os estudos de citação no campo da Cancerologia e contribuir para o avanço do entendimento da Ciência da Informação acerca do tema. O trabalho investiga o comportamento de citação dos docentes-pesquisadores no campo da Cancerologia brasileira, partindo da seguinte questão de pesquisa: como se caracterizam os padrões de comportamento de citação dos docentes-pesquisadores no campo da Cancerologia brasileira? Com base nessa questão, delineou-se como objetivo caracterizar o comportamento de citação dos docentespesquisadores do campo da Cancerologia brasileira, através da análise das referências elencadas por estes em suas produções científicas publicadas no período de 2005-2015.

\section{INSTITUCIONALIZAÇÃO DO CAMPO DA CANCEROLOGIA BRA- SILEIRA}

No Brasil, os primeiros indícios de interesse científico dos médicos pela temática câncer surgiu a partir de publicações e discussões em eventos internacionais no início do século XX (TEIXEIRA, 2009). De acordo com o autor,

[...] o fazer técnico-científico se mistura com a busca de reconhecimento profissional, em nosso caso, exemplificada na escolha de objetos de estudos valorizados pelas ciências médicas dos países centrais. (TEIXEIRA, 2009, p. 104105).

Do ponto de vista científico, este cenário pode ser explicado por dois aspectos. Por um lado, um tipo de subserviência cultural, por parte dos médicos brasileiros, aos objetos de investigações dos seus pares de países mais desenvolvidos, modus operandi ainda identificado nos dias atuais no campo científico; por outro, o reflexo do retardo por parte do governo em institucionalizar a 
pós-graduação brasileira. Visto assim, é compreensível o atraso na institucionalização da pós-graduação no campo da Cancerologia brasileira, fato que só ocorreu em 1987, com o credenciamento do primeiro programa de pós-graduação em Oncologia, da Universidade de São Paulo. Dez anos após o credenciamento do programa da Universidade de São Paulo, a Fundação Antônio Prudente - Hospital A. C. Camargo, também sediada no estado de São Paulo, credenciou um programa no campo da Cancerologia.

Em pesquisa realizada na Plataforma Sucupira, constatou-se que existiam, até janeiro de 2017, oito programas de pósgraduação no campo, sendo que sete se encontravam em funcionamento e um em projeto (COORDENAÇÃO DE APERFEIÇOAMENTO DE PESSOAL DE NÍVEL SUPERIOR, 2017), entre eles, Programa de pós-graduação da Universidade de São Paulo (PPG-USP) $^{3}$ - 1987; Programa de pósgraduação da Fundação Antônio Prudente Hospital A. C. Camargo (PPG-FAP) - 1997; Programa de pós-graduação do Instituto Nacional de Câncer (PPG-INCA) - 2005; Programa de pós-graduação da Fundação Pio XII - Hospital de Câncer de Barretos (PPG-HCB) - 2011; Programa de pósgraduação da Universidade Federal do Pará (PPG-HCB) - 2011; Programa de pósgraduação da Universidade de São Paulo/Ribeirão Preto (PPG-USP/RP) - 2012 e Programa de pós-graduação da Universidade Estadual de Campinas (PPG-UNICAMP) 2016. O cenário geográfico dos programas de pós-graduação no campo da Cancerologia brasileira é, quase em sua totalidade, centralizado na região Sudeste, destacando-se o estado de São Paulo, com $6(75,0 \%)$ programas de pós-graduação. Deste total localizado no estado de São Paulo, cinco programas estão credenciados e um em projeto.

Acerca das linhas de pesquisas, no campo da Cancerologia, o documento de área reforça o papel de diretriz que as linhas

\footnotetext{
3 Neste artigo, optou-se em empregar código alfabético, elaborado pelas autoras para identificar os programas de pós-graduação no campo da Cancerologia.
}

de pesquisa desempenham no campo científico, afirmando que as linhas de pesquisa "[...] devem estar vinculadas à proposta geral do curso.” (COORDENAÇÃO DE APERFEIÇOAMENTO DE PESSOAL DE NÍVEL SUPERIOR, 2012, p. 12). Em cada programa de pós-graduação, as divisões, mesmo que tênues, geradas a partir das linhas de pesquisa, não fundamentam uma divisão entre seus docentes-pesquisadores e discentes, mas sim uma delimitação no escopo dos estudos e investigações. Entre os programas de pós-graduação no campo da Cancerologia brasileira, identificaram-se 50 linhas de pesquisa vigentes e 37 desativadas 37.

Apontados como um dos contributos para a institucionalização de um campo científico, os periódicos científicos são imprescindíveis na disseminação dos resultados das pesquisas entre os pesquisadores dos diversos campos científicos. No campo da Cancerologia brasileira, apesar de os pesquisadores utilizarem também outros canais de comunicação, como apresentação oral em eventos científicos, livros, capítulo de livros e anais de congressos, os artigos publicados em periódicos científicos predominam como principal meio de publicação e comunicação no campo. Isto é evidente, considerando que o periódico científico apresenta a característica de ser uma publicação rápida, pois a otimização das etapas do processo editorial do periódico científico, em comparação com outros canais de comunicação como o livro, é menos lenta e mais acessível. Associadas a estes aspectos estão as orientações descritas no documento de área da Capes do campo da Cancerologia, as quais elegem os artigos publicados em periódicos científicos como um dos principais indicadores da produção intelectual nos programas de pós-graduação do campo (COORDENAÇÃO DE APERFEIÇOAMENTO DE PESSOAL DE NÍVEL SUPERIOR, 2009, 2012, 2016).

A relevância atribuída ao artigo publicado em periódico científico no campo da Cancerologia brasileira é evidenciada também na quantidade de títulos de periódicos indexados na especialidade Médica Oncologia, na Biblioteca Virtual em Saúde (BVS). 
Do total de 306 títulos de periódicos científicos indexados na BVS, 218 títulos são correntes. Do total de títulos correntes, 212 são estrangeiros e seis nacionais, revelando dois aspectos: em primeiro lugar, um amplo leque de opções de títulos de periódicos para publicação dos resultados das pesquisas, reafirmando o prestígio atribuído a este canal de comunicação no campo da Cancerologia. Em segundo lugar, revela um número de títulos de periódicos nacionais que, apesar de diminuto em comparação com os títulos de periódicos estrangeiros, mostra-se equilibrado, quando consideramos que há somente sete programas de pós-graduação no campo da Cancerologia brasileira.

\section{COMPORTAMENTO DE CITAÇÃO}

Assim como os canais de comunicação variam em conformidade com as especificidades do campo científico, o mesmo observa-se em relação às características do comportamento de citação. Em pesquisa realizada em 270 artigos, selecionados aleatoriamente nos campos da Administração, Avaliação Educacional e Psicologia Clínica, em um período de dez anos, Smart e Bayer (1986) identificaram que trabalhos em autoria múltipla alcançam uma taxa de citação mais elevada que os de autoria individual. Segundo Bufrem (2009), esse comportamento

[...] pode representar uma tendência já verificada na literatura mundial, a de estar a ciência cada vez mais sendo desenvolvida em equipe [...] principalmente pelas possibilidades de intercâmbios interdepartamentais e interinstitucionais $[. .$.$] .$

Essa tendência pode também estar relacionada à própria característica do objeto de pesquisa de alguns campos científicos, os quais possuem alcance que transcendem os departamentos e instituições e, assim, a pesquisa colaborativa é um recurso utilizado.

Lancaster, Lee e Diluvio (1990) apresentaram, em um artigo, resultados de duas pesquisas independentes realizadas nos campos da Matemática coreana e da Litera- tura filipina. Nesse trabalho, os autores identificaram que "[...] o lugar da publicação pode exercer alguma influência sobre o comportamento da citação." (LANCASTER; LEE; DILUVIO, 1990, p. 239, tradução nossa). A variação no tipo de canal a ser publicado - como anais de evento, artigo de periódicos, livros, entre outros - pode apresentar relação com fatores como o fluxo de informação em um campo científico. Os campos mais dinâmicos tendem a recorrer ao artigo de periódico para publicar os resultados da pesquisa e consequentemente acabam sendo os mais citados.

Em 2016, Nishy, Rana e Mini examinaram artigos publicados no período de 2004-2008, por pesquisadores indianos do National Institute for Interdisciplinary Science and Technology (NIIST), indexados na Web of Science (WoS). De acordo com os autores, entre as características presentes nas citações destes artigos, estão: periódicos com alto fator de impacto; presença de insularidade e $68 \%$ das citações de publicações dos últimos 10 anos.

Com o objetivo de analisar a tipologia da fonte citada pelos pesquisadores das oito áreas definidas pela Scientific Electronic Library Online (SciELO), Mugnaini, Meneghini e Packer (2007) realizaram um levantamento em 133 periódicos indexados nessa base de dados em 2004. Nessa pesquisa, os autores constataram que nas Ciências da Vida a principal fonte de citação são os artigos científicos.

Skilton (2009), por sua vez, investigou o tipo de autoria nos artigos das Ciências Naturais. Segundo o autor, as citações neste campo são resultantes de

[...] artigos em coautoria, com equipes incluindo pesquisadores frequentemente citados e equipes cujos membros têm experiências disciplinares diversas. (SKILTON, 2009 , p. 525, tradução nossa).

\footnotetext{
4 No campo científico, insularidade é a predominância de citações de produções do país de origem do pesquisador-autor em detrimento de produções internacionais (LADLE; TODD; MALHADO, 2012).
} 
Vinther e Rosenberg realizaram pesquisa com títulos de periódicos classificados como de Medicina Geral e Interna no Journal Citation Reports (JCR), publicados no período de 2001-2010, e constataram que o idioma influencia na frequência de citação de uma publicação. Os autores explicam que "publicar em inglês aumenta o número de citações e, portanto, o fator de impacto [...]" (VINTHER; ROSENBERG, 2012, p. 4).

Lira et al. (2013), por sua vez, investigaram os periódicos Arquivos Brasileiros de Oftalmologia e a Revista Brasileira de Oftalmologia, a fim de verificar a relação entre o número de vezes que um artigo foi citado e a língua deste. Os autores concluíram que as citações de artigos publicados em língua inglesa foram mais elevadas, em comparação com o número de citações dos artigos em português.

Em artigo publicado em 2001, Van Dalen e Henkens descrevem uma pesquisa realizada com 17 títulos de periódicos do campo da Demografia, publicados entre 1990 e 1992, indexados no Social Science Citation Index (SSCI). Segundo os autores, a representatividade do título de periódico e o idioma são fatores que influenciam o ato de citar, em especial se estiver escrito em língua inglesa. Sobre o título de periódico, é importante lembrar que o ato de publicar os resultados da pesquisa representa para o pesquisador uma regra com particularidades para cada campo. O campo, ao mesmo tempo em que oferece para os pesquisadores uma variedade de canais, aponta os mais expressivos.

Em 2015, Thornley et al. investigaram a representatividade do título de periódico no impacto das citações e publicaram o resultado da investigação realizada com 87 pesquisadores do Reino Unido e dos Estados Unidos. Os autores comprovaram que a "autoridade e a confiabilidade da fonte citada", que envolvem, entre outros aspectos, a autoria da publicação, o título de periódico em que foi publicada a produção e o grupo de pesquisa são fatores que influenciam na escolha da fonte a ser citada.

\section{PROCEDIMENTOS METODOLÓ- GICOS}

A pesquisa é descritiva e de abordagem quantitativa. O corpus da pesquisa foi composto pelas citações presentes nas produções dos docentes-pesquisadores de seis programas de pós-graduação no campo da Cancerologia brasileira, que oferecem Mestrado Acadêmico e Doutorado: PPG-FAP; PPG-HCB; PPG-INCA; PPG-UFPA; PPGUSP e PPG-USP/RP.

Com base nas produções de 245 docentes-pesquisadores credenciados nos programas de pós-graduação, realizou-se uma análise de 1.016 citações. Selecionaramse as citações que obtiveram frequência igual ou maior a $8(\geq 8)$.

Importante sublinhar que essas produções se encontravam elencadas no campo "Artigos completos publicados em periódicos", do Currículo Lattes, publicadas no período 2005-2015 e indexadas na WoS. A coleta de dados ocorreu em quatro etapas: a) coleta e lançamento dos dados referentes às produções dos docentes-pesquisadores em um banco de dados no Microsoft Excel; b) localização e migração dos dados das produções da WoS para o HistCite $^{\mathrm{TM}}$, versão 12.03.17 $7^{5}$, criando assim o banco de dados das produções; c) identificação das referências citadas nas produções dos docentespesquisadores; e d) localização e migração dos dados das citações da WoS para o HistCite ${ }^{\mathrm{TM}}$, criando assim o banco de dados das citações.

\section{ANÁLISE E INTERPRETAÇÃO DOS RESULTADOS}

Apresentam-se, nesta seção, alguns dos padrões de comportamento de citação encontrados no campo da Cancerologia brasileira, a saber: tipologia da fonte citada; tipo de autoria; idioma; ano de publicação; insularidade e títulos de periódicos mais citados.

Os docentes-pesquisadores da Cancerologia brasileira apresentam variados ti-

\footnotetext{
5 Software livre, disponível em:

$<$ http://interest.science.thomsonreuters.com/forms/ HistCite $/>$.
} 
pos de produção científica e técnica. As in-

das estão reunidas no Gráfico 1.

formações sobre a tipologia das fontes cita-

Gráfico 1 - Tipologia das produções citadas pelos docentes-pesquisadores (2005-2015)

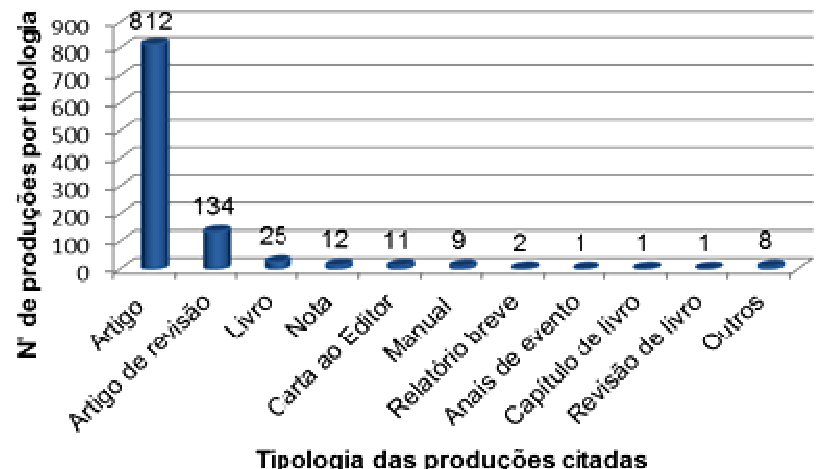

Fonte: Dados da pesquisa (2017).

Os resultados mostraram que, entre os docentes-pesquisadores no campo da Cancerologia brasileira, $812 \quad(80,0 \%)$ "Artigos" foram citados, revelando a predominância desta tipologia nas ocorrências das produções citadas. Este resultado, além de sugerir uma forte aproximação dos resultados encontrados por Mugnaini, Meneghini e Packer (2007), nas Ciências da Vida, ao investigar 133 periódicos indexados na base de dados SciELO em 2004, indica que o artigo deve ser o principal canal de comunicação entre os docentes-pesquisadores do campo (COORDENAÇÃO DE APERFEIÇOAMENTO DE PESSOAL DE NÍVEL SUPERIOR, 2009, 2012, 2016). Em relação à tipologia "Artigo de revisão", embora encontre-se como a segunda tipologia mais citada pelos docentes-pesquisadores, sua ocorrência corresponde a $134 \quad(13,2 \%)$ citações, bem abaixo da primeira tipologia.

6 Para análise das tipologias das produções citadas pelos docentes-pesquisadores, a categoria "Artigo" refere-se a "parte de uma publicação com autoria declarada, que apresenta e discute ideias, métodos, técnicas, processos e resultados nas diversas áreas do conhecimento", podendo ser de temáticas ou abordagens originais, enquanto que a categoria "Artigo de revisão" destina-se a "parte de uma publicação que resume, analisa e discute informações já publicadas." (ASSOCIAÇÃO BRASILEIRA DE NORMAS TÉCNICAS, 2003, p. 2).
"Anais de evento", "capítulo de livro" e "revisão de livro" representam as tipologias com baixa ocorrência, com $1(0,1 \%)$ citação cada uma. No item "outros" foram agrupadas as citações de $6(0,6 \%)$ "softwares", $1(0,1 \%)$ "editorial" e $1(0,1 \%)$ "notícia".

Um segundo padrão observado foi o tipo de autoria das citações, se individual ou múltipla. A distribuição dessa tipologia encontra-se disposta no Gráfico 2.

As produções citadas com autoria individual são diminutas entre as publicações dos docentes-pesquisadores no campo da Cancerologia brasileira. Pode-se notar que 80 $(7,9 \%)$ das produções citadas eram de autoria individual. A presença de autoria múltipla entre as produções citadas representou 92,1\%, ou seja, 936 publicações. Resultados semelhantes foram encontrados na pesquisa de Smart e Bayer (1986) no campo da Administração e de Skilton (2009) nas Ciências Naturais. Esse padrão de comportamento de citação no campo da Cancerologia brasileira quanto ao tipo de autoria pode estar associado ao crescimento no número de programas de pós-graduação e, consequentemente, de pesquisadores no campo. Observou-se que entre 1997 e 2012 foram credenciados cinco programas. Além disso, este comportamento de citação pode estar relacionado também a uma tendência observada por Bufrem (2009): a realização de pesquisas em equipe. 
Gráfico 2 - Disposição dos tipos de autoria das produções citadas pelos docentes-pesquisadores por ano (2005-2015)

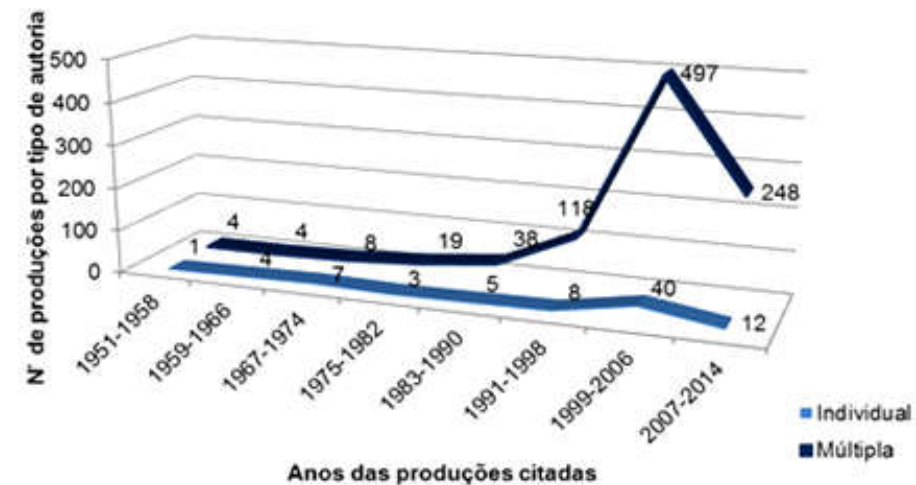

Fonte: Dados da pesquisa (2017).

Um terceiro padrão analisado foi o idioma das produções científicas citadas. Os resultados evidenciaram que 1005 (99,0\%) das citações presentes nas produções dos docentes-pesquisadores no campo da Cancerologia brasileira foram de produções escritas em Inglês. As pesquisas de Van Dalen e Henkens (2001), Vinther e Rosenberg (2012) e Lira et al. (2013) também evidenciaram a presença de citações em língua inglesa nas publicações investigadas. A predominância de citações em Inglês parece justificar-se no fato de que as produções escritas nesta língua alcançam maior visibilidade junto aos docentes- pesquisadores do campo científico e consequentemente são mais propensas a serem citadas. Lira et al. (2013) ainda esclarecem que, no caso dos artigos científicos, não é suficiente escrever em língua inglesa apenas os resumos e palavraschave, mas todo o artigo.

Um quarto padrão de comportamento foi o ano de publicação da citação. Para analisar as citações na categoria ano de publicação, decidiu-se por agrupar as produções citadas em oito intervalos, de oito anos cada. No Gráfico 3 é possível examinar o número de ocorrência das citações por intervalos.

\section{Gráfico 3 - Ano de publicação das produções citadas pelos} docentes-pesquisadores (2005-2015)

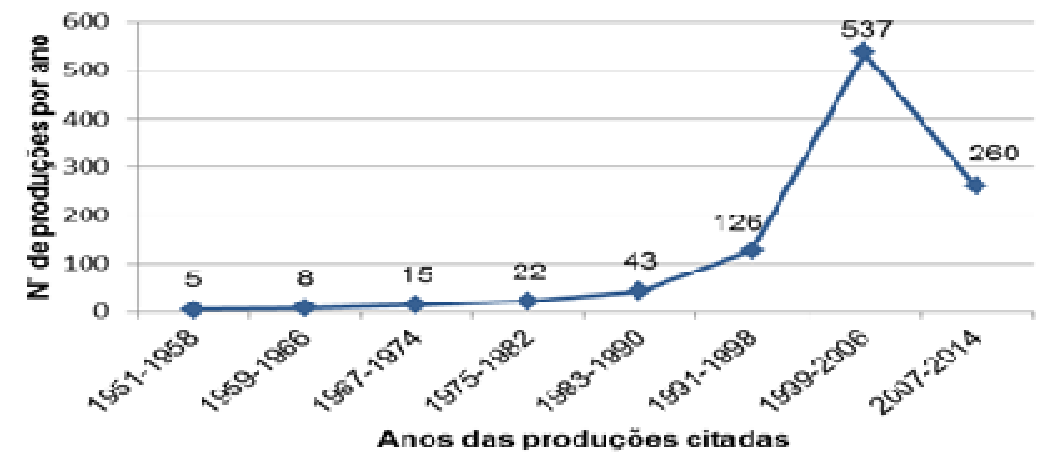

Fonte: Dados da pesquisa (2017).

Entre as produções dos docentespesquisadores no campo da Cancerologia brasileira, publicadas no período 2005-2015, identificou-se que, apesar da ocorrência de $219(21,5 \%)$ citações referentes ao período de 1951 a 1998, as citações mais recentes são 
predominantes, correspondendo a 797 $(78,5 \%)$ citações. Ao comparar os intervalos 1999-2006 e 2007-2014, registrou-se um declínio na quantidade de citações presentes nas produções dos docentes-pesquisadores com o ano de publicação referente ao segundo intervalo.

A partir dessas constatações, é possível inferir que a Cancerologia é um campo dinâmico que demanda informações atualizadas e que o declínio no número de citações registrado no segundo período pode estar associado à proximidade com $\mathrm{O}$ período em que as publicações dos docentes-pesquisadores foram analisadas. Nishy, Rana e Mini (2016), ao investigar pesquisadores do NIIST, também constataram semelhante característica nas citações destes e justificaram este comportamento de citação pelas realizações de pesquisas contemporâneas.

$\mathrm{Na}$ literatura científica, observa-se que os estudos de citações vêm investigando a presença da insularidade nas publicações de diferentes campos, como observado nas pesquisas de Nishy, Rana e Mini (2016), com pesquisadores do NIIST, e de Campos et al. (2016) no campo da Etnobiologia e na Etnozoologia. Um quinto padrão observado no Campo da Cancerologia também foi a insularidade. Quando cotejada a presença de autores brasileiros nas citações das publicações dos docentes-pesquisadores no campo da Cancerologia brasileira, nota-se que, entre os 16 países de origem dos autores citados, a ocorrência de autores brasileiros é de pequena dimensão, correspondendo a $290(24,2 \%)$ autores brasileiros citados. Os autores mais citados são provenientes dos Estados Unidos da América e contabilizam $451(38,0 \%)$ autores citados. Esclarece-se que a representatividade de autores brasileiros entre os 16 autores mais citados não representa insularidade no campo. O baixo nível de insularidade nas citações presentes nas produções dos docentes-pesquisadores no campo da Cancerologia brasileira pode apontar um nível de amadurecimento do campo.

Quadro 1 - Títulos de periódicos mais citadas pelos docentes-pesquisadores (2005-2015)

\begin{tabular}{|c|l|c|c|}
\hline \multicolumn{1}{|c|}{ Tátulos de periódicos citados } & Quant. citações & $\begin{array}{c}\text { Qualis/ } \\
\text { Capes } \\
\text { (2013-2016) }\end{array}$ \\
\hline $1^{\circ}$ & New England Journal of Medicine & 47 & A1 \\
\hline $2^{\circ}$ & Proceedings of the National Academy of Sciences of the United States of America & 38 & A1 \\
\hline $3^{\circ}$ & Cancer Research & 26 & A1 \\
\hline $4^{\circ}$ & Science & 24 & A1 \\
\hline $5^{\circ}$ & Nature & 23 & A1 \\
\hline $6^{\circ}$ & Blood & 22 & A1 \\
\hline $7^{\circ}$ & Journal of Clinical Oncology & 21 & A1 \\
\hline $8^{\circ}$ & Cell & 17 & A1 \\
\hline $9^{\circ}$ & International Journal of Cancer & 17 & A1 \\
\hline $10^{\circ}$ & Journal of the National Cancer Institute & 17 & A1 \\
\hline $11^{\circ}$ & Lancet & 17 & A1 \\
\hline $12^{\circ}$ & Nucleic Acids Research & 14 & A1 \\
\hline $13^{\circ}$ & Nature Reviews. Cancer & 13 & Outros \\
\hline $14^{\circ}$ & Bioinformatics & 12 & Aampos \\
\hline $15^{\circ}$ & CA-A Cancer Journal for Clinicians & 12 & Sem \\
\hline $16^{\circ}$ & Cancer Epidemiology Biomarkers \& Prevention & A2 \\
\hline Fonte: Dados da pesquisa (2017). & classificaça \\
\hline
\end{tabular}


Um sexto padrão de comportamento de citação observado no campo em análise foi títulos de periódicos. A análise dos títulos de periódicos científicos mais citados mostrou alta representatividade dos títulos de periódicos estrangeiros no ranking dos 16 mais citados pelos docentes-pesquisadores do campo da Cancerologia brasileira. O Quadro 1 dispõe detalhes sobre esta distribuição.

Referente aos 16 títulos de periódicos mais recorrentes nas citações das publicações dos docentes-pesquisadores do campo da Cancerologia brasileira, 13 $(81,3 \%)$ títulos de periódicos foram classificados no estrato A1, em Medicina I e um título de periódico, o Nature Reviews Cancer, foi classificado no estrato A1 em Ciências Ambientais; Geografia; Medicina II e Medicina Veterinária, todos A1, totalizando 14 títulos de periódicos com estrato A1. A classificação dos títulos de periódicos mais recorrentes nas citações no estrato A1, o qual é considerado mais alto no Qualis/Capes, reafirma os resultados das pesquisas realizadas por Van Darlei e Henkens (2001) e Thornley et al. (2015), em que se constata que a qualidade do título de periódico influencia no comportamento de citação dos pesquisadores.

\section{CONSIDERAÇÕES FINAIS}

Mais que nunca, o fazer científico está intrinsecamente associado ao ato de tornar públicos os resultados da pesquisa. No campo científico, a publicação tem um papel importante no compartilhamento e na avaliação dos resultados de uma pesquisa, podendo constituir-se em fonte de pesquisa para outros pesquisadores. $\mathrm{O}$ ato de tornar públicos os resultados de uma pesquisa, porém, não garante que esta seja citada em textos posteriores. Observaram-se no campo da Cancerologia brasileira alguns padrões quanto ao comportamento de citação. Citase predominantemente: em língua inglesa; em autoria múltipla; artigo científico; produções recentes; artigos publicados em periódicos com Qualis/Capes A1, A2 e B1 do período de 2013-2016; autores estrangeiros. Estes padrões de comportamento sugerem que a Cancerologia brasileira é um campo em consolidação e que os docentes-pesquisadores recorrem às citações que lhes possibilitem alcançar reconhecimento de seus pares em âmbito internacional.

A partir dos resultados apresentados no presente artigo, constata-se que o ato de citar, além de constituir uma ação necessária para a construção do conhecimento científico, pode estar associado a uma escolha mais ou menos consciente, no sentido de que o docente-pesquisador alcance visibilidade e reconhecimento dos pares.

Sugere-se que novas pesquisas sejam realizadas para acompanhar possíveis mudanças nas características do comportamento de citação dos docentespesquisadores do campo da Cancerologia brasileira.

\title{
CITATION BEHAVIOR IN THE FIELD OF BRAZILIAN CANCEROLOGY
}

\begin{abstract}
This paper aims at characterizing the citation behavior of the professors-researchers in the field of Brazilian Cancerology, through the analysis of the references listed in their scientific productions published in the period 2005-2015. The sample consisted of 1,016 citations. Results suggest that in the field under analysis, the article is the main typology of the productions cited among the professors-researchers, corresponding to $80 \%$ of the citations. The observed citation behavior in relation to multiple authorship is reproduced thronghout the analyzed period, with a higher occurrence than that of individual authorship, 936 (92, 1\%) and 80 (7, 9\%), respectively. Regarding the language of the citations, English corresponds to 1005 $(99,0 \%)$ of the citations.
\end{abstract}

Keywords

Citation analysis. Brazilian Cancerology. Citation behavior. Artigo recebido em 04/11/2017 e aceito para publicação em 20/06/2018 


\section{REFERÊNCIAS}

ASSOCIAÇÃO BRASILEIRA DE NORMAS TÉCNICAS. NBR 6022: informação e documentação: artigo em publicação periódica científica impressa: apresentação. Rio de Janeiro, 2003.

BUFREM, L. S. Relações interinstitucionais e autoria em artigos de revistas científicas de Ciência da Informação no Brasil. In: ENCONTRO NACIONAL DE PESQUISA EM CIÊNCIA DA INFORMAÇÃO, 10., 2009, João Pessoa, PB. Anais eletrônicos... João Pessoa: UFPB, 2009. Disponível em: $<$ http://enancib.ibict.br/index.php/enancib /xenancib/paper/viewFile/3299/2425>. Acesso em: 25 out. 2016.

CAMPOS, J. L. A. et al. Insularity and citation behavior of scientific articles in young fields: the case of etnobiology. Scientometrics, Amsterdam, v. 109, p. 1037-1055, 2016. Disponível em: $<$ https://link.springer.com/content/pdf/10 .1007\%2Fs11192-016-2067-2.pdf>. Acesso em: 15 fev. 2017

CONSELHO NACIONAL DE DESENVOLVIMENTO CIENTÍFICO E TECNOLÓGICO. 2017. Disponível em: $<$ https://sucupira.capes.gov.br/sucupira/>. Acesso em: 15 jan. 2017.

\section{Documento de área Medicina I.}

2009. Disponível em: $<$ http://capes.gov.br/images/stories/downl oad/avaliacao/MED_I20nov09q.pdf>.

Acesso em: 05 ago. 2012.

\section{Documento de área Medicina I.}

2012. Disponível em:

$<$ http://www.capes.gov.br/images/stories/ downlo-

ad/avaliacaotrienal/Docs_de_area/M edicina_I_doc_area_e_comiss $\%$ C3\%A3o_att08d eoutubro.pdf>. Acesso em: 05 ago. 2014.

\section{Documento de área Medicina I.}

2016. Disponível em:

$<$ http://capes.gov.br/images/documentos/
Documen-

tos_de_area_2017/15_MED_I_d ocarea_2016.pdf $>$. Acesso em: 05 ago. 2016.

FREIRE, I. M. O desviante secreto: um exercício conceitual. Ciência da Informação, Brasília, DF, v. 25, n. 3, p. 1-17, dez. 1996. Disponível em:

$<$ http://revista.ibict.br/ciinf/article/view/6 42/646>. Acesso em: 14 nov. 2014

GARVEY, W. D. Communication: the essence of Science. Oxford: Pergamon, 1979.

LADLE, R. J.; TOOD, P. A.; MALHADO, A. C. M. Assessing insularity in global Science. Scientometrics, Amsterdam, v. 93, p. 745-750, 2012. Disponível em: $<$ https://link.springer.com/content/pdf/10 .1007\%2Fs11192-012-0703-z.pdf>. Acesso em: 14 mar. 2015.

LANCASTER, F. W.; LEE, S.-Y. K.; DILUVIO, C. Does place of publication influence citation behavior? Scientometrics, Amsterdam, v. 19, n. 3-4, p. 239-244, 1990. Disponível em:

$<$ https://link.springer.com/content/pdf/10 $.1007 \% 2 F B F 02095350 . p d f>$. Acesso em 15 mar. 2016.

LIRA, R. P. C. et al. Influence of English language in the number of citations of articles published in Brazilian journals of ophthalmology. Arquivo Brasileiro de Oftalmologia, São Paulo, v. 76, n. 1, p. 26-28, 2013. Disponível em:

<http://www.scielo.br/pdf/abo/v76n1/a0 8v76n1.pdf>. Acesso em: 05 out. 2015.

MEADOWS, A. J. A comunicação científica. Tradução de Antonio Agenor Briquet de Lemos. Brasília, DF: Briquet de Lemos, 1999.

MUELLER, S. P. M. Literatura científica, comunicação científica. In : TOUTAIN, L. M. B. B. (Org.). Para entender a Ciência 
da Informação. Salvador: EDUFBA, 2007. (Saladeaula; 6).

MUGNAINI, R.; MENEGHINI, R.; PACKER, A. Citation profiles in Brasilian journals of the SciELO databasse in different scientific areas. In: INTERNATIONAL CONFERENCE OF THE INTERNATIONAL SOCIETY FOR SCIENTOMETRICS AND INFORMTRICS, 11., 2007, Madrid. Proceedings... Madrid, 2007. Disponível em :

$<$ https://www.researchgate.net/publication /275274284_Citation_profiles_in_Brazilian_ jour-

nals_of_the_SciELO_database_in_different _scientific_areas>. Acesso em : $15 \mathrm{dez}$. 2015.

NIEDER, C. et al. Scientific impact of studies published in temporarily available radiation oncology journasl: a citation analysis. SpringerPlus, [S.l.], v. 4, n. 93, p. 2-6, 2015. Disponível em:

$<$ https://www.ncbi.nlm.nih.gov/pmc/articl es/PMC4348359/

pdf/40064_2015_Article_885.pdf>. Acesso em: 14 mar. 2016.

NISHY, P; RANA, M. S.; MINI, S. Citations behaviour of Indian scientists from an interdisciplinary research institute: A case study of CSIR-NIIST. Annals of Library and Information Studies, Nova Deli, v. 63, p. 68-73, 2016. Disponível em: <http://nopr.niscair.res.in/bitstream/12345 6789/33891/1/ALIS $\% 2063 \% 281 \% 29 \% 206$ 8-73.pdf>. Acesso em: 10 jan. 2016.

SKILTON, P. F. Does the human capital of teams of natural Science authors predict citation frequency? Scientometrics, Budapest, v. 78, n. 3, p. 525-542, 2009. Disponível em:

$<$ https://link.springer.com/content/pdf/10 .1007\%2Fs11192-007-1953-z.pdf>. Acesso em: 09 out. 2015.
SMART, J. C.; BAYER, A. E. Author collaboration and impact: a note on citation rates of single and multiple authored articles. Scientometrics, Budapest, v. 10, n. 5-6, p. 297305, 1986. Disponível em:

$<$ https://link.springer.com/content/pdf/10 $.1007 \% 2 F B F 02016776 . p d f>$. Acesso em: 03 out. 2015.

STUMPF, I. R. C. Passado e futuro das revistas científicas. Ciência da Informação, Brasília, DF, v. 25, n. 3, 1996. Disponível em:

$<$ http://revista.ibict.br/ciinf/article/viewFil e/463/422>. Acesso em: 20 jun. 2006.

TEIXEIRA, L. A. O câncer na mira da medicina brasileira. Revista Brasileira de História da Ciência, Rio de Janeiro, v. 2, n. 1, p. 104-117, jan.-jun. 2009. Disponível em: $<$ http://www.historiadocancer.coc.fiocruz.b r/images/site/pdfs/luiz_cancermira.pdf $>$. Acesso em: 14 mar. 2016.

THORNLEY, C. et al. The role of trust and authority in the citation behaviour of researchers. Informationresearch, Boras, v. 20, n. 3, sep. 2015. Disponível em:

$<$ https://researchoutput.csu.edu.au/ws/por talfiles/portal/9366265>. Acesso em 23 ago. 2016.

VAN DALEN, H. P.; HENKENS, K. What makes a scientific article influential? The case of demographers. Scientometrics, Budapest, v. 50, n. 3, p. 455-482, 2001. Disponível em:

$<$ https://link.springer.com/content/pdf/10 $.1023 \% 2 \mathrm{FA} \% 3 \mathrm{~A} 1010510831718 . \mathrm{pdf}>$.

Acesso em: 10 out. 2015.

VINTHER, S.; ROSENBERG, J. Impact factor trends for general medical journals: non-english-language journals are lagging behind. Swiss Medical Weekly, [S.l.], v. 142, p. w13572, 2012. 